\title{
Implementación de una red inalámbrica de sensores para la gestión de luminarias utilizando IPv6
}

\author{
(Implementation of a Wireless Sensor Network \\ for Electric Light Bulb Management Using IPv6)
}

\author{
Carlos Egas ${ }^{1}$, Darío Viracocha ${ }^{1}$, Juan Rivera ${ }^{2}$
}

\begin{abstract}
Resumen
Se presentan los resultados de la implementación del prototipo de red inalámbrica de sensores para gestionar las luminarias en los hogares mediante el uso de los nodos de sensores Iris, diseñados para funcionar con batería durante largos períodos de tiempo y con capacidades de procesamiento limitadas. Los nodos usan direcciones 6LoWPAN; la conversión de la dirección a IPv6 se hace por medio de un gateway. Las luminarias se gestionan por medio de dispositivos conectados a la red Ipv6, con sistema operativo Android. El correcto funcionamiento del prototipo se demuestra al validar las funcionalidades del sistema de gestión implementado.
\end{abstract}

Palabras clave

Domótica, WSN, 6LoWPAN, TinyOS, IEEE 802.15.4.5.

\begin{abstract}
In this study the results of the implementation of the wireless sensor network prototype, to manage the luminaires in homes are presented, using Iris sensors nodes, designed to work with battery for long periods of time and with limited processing capabilities. The nodes use 6LoWPAN addresses. The address conversion to IPV6 is done by a gateway. The luminaires are managed using devices connected to IPV6 network, with Android operating system. The correct functioning of the prototype is demonstrated by validating the functionalities of the management system implemented.
\end{abstract}

\section{Keywords}

Domotic, WSN, 6LOWPAN, TinyOS, IEEE 802.15.4.5.

\section{Introducción}

En la actualidad, la iluminación inteligente es un área fundamental en el desarrollo de hogares inteligentes, ya que permite la administración del consumo de energía y la gestión remota utilizando dispositivos portátiles.

Un número creciente de fabricantes implementan soluciones en la industria de la iluminación inteligente empleando diferentes protocolos de comunicaciones como KNX (Han, Wu y Tang, 2010), BACnet (Park y Hong, 2010), DALI (Wohlers, Andonov y Gunnar, 2013), ZigBee-ZHA /ZBA (Domínguez, Touhafi y Tiete, 2012), lo cual crea problemas relacionados con la baja compatibilidad y extensibilidad, debido a que los productos generados no pueden interactuar entre sí; por lo que es necesario proponer soluciones de iluminación inteligente que permitan una conectividad global utilizando la red internet.

Una de las tecnologías empleadas en el Internet de las Cosas son las redes inalámbricas de sensores (WSN, por sus siglas en inglés) (Singh, 2016), que se caracterizan porque los nodos están conformados por microcontroladores que tienen una baja capacidad de procesamiento, operan con baterías, a bajas velocidades de transmisión y son diseñadas para estar operativas

1 Escuela Politécnica Nacional (EPN). Quito, Ecuador (carlos.egas@epn.edu.ec, dario.viracocha@epn.edu.ec).

2 Universidad UTE. Quito, Ecuador (jcrivera@ute.edu.ec) 
por largos períodos de tiempo. La diferencia principal con nodos basados en Arduino (microcontrolador) y un Raspberry Pi (microprocesador) son las siguientes: las capacidades de entradas y salidas, el rendimiento de la unidad central de procesamiento (CPU, por sus siglas en inglés), el consumo de energía y su diseño para recibir energía de la red eléctrica. Si bien Arduino también emplea procesadores de 8 bits y el Raspberry Pi, 8 y 32 bits (ATmega 328, ATmega 2560, Atmel SAM3X8E); los nodos sensores de una red WSN consumen menos energía.

El protocolo 6LoWPAN (Al-Kashoash, 2017) fue diseñado para ejecutarse en nodos de redes inalámbricas de sensores (Sohraby, Minoli y Znati, 2007) y permitir su compatibilidad con la red internet con IPv6 utilizando un nodo pasarela (gateway, en inglés). Se puede usar 6LoWPAN en un nodo sensor basado en Arduino o Raspberry Pi; sin embargo, estos nodos tienen los recursos para operar con el protocolo IPv6 y 802.11 (WiFi, por sus siglas en inglés). En aplicaciones donde el requerimiento es la operación por largos períodos de tiempo, usando únicamente baterías sin recargarlas, la utilización de estas plataformas no es óptima.

La imposibilidad de implementar la pila de protocolos de internet en un nodo sensor que opera con el estándar 802.15.4 (IEEE, 2011), entre ellos IPV6, ha dado lugar al desarrollo de los protocolos 6LoWPAN y ZigBee. El protocolo 6LoWPAN, que utilizan las redes de sensores multisalto, es fundamental para el desarrollo de nuevas aplicaciones en el Internet de las Cosas, más aún cuando el grado de penetración de IPv6 en la red Internet está creciendo exponencialmente.

Si bien en la actualidad existen aplicaciones para el monitoreo, por ejemplo, de signos vitales, monitoreo ambiental, medición inteligente, domótica e incluso automatización industrial; muchas de ellas trabajan con protocolos propietarios de los vendedores, por lo que en la mayoría de los casos no posibilitan la conectividad directa con otras tecnologías.

La utilización de 6LoWPAN permite implementar nuevas aplicaciones y protocolos de servicios, e integrar dispositivos portátiles con bajas capacidades de procesamiento, debido a que posibilita la conectividad de extremo a extremo con direcciones IP públicas asignadas a los nodos fuente y destino. El desafío con la utilización del protocolo 6LoWPAN para soluciones de control de iluminación se debe a que actualmente no hay un protocolo estándar en la capa aplicación de la arquitectura de la red inalámbrica de sensores (Rand, 2018). Esto significa que, aunque la conectividad en la red está bien definida y se basa en estándares abiertos, por el momento no se han desarrollado soluciones basadas en estándares de la capa aplicación.

El protocolo 6LoWPAN se ha usado de manera exitosa en la gestión de luminarias en diferentes aplicaciones, tales como en un sistema de iluminación inteligente basados con topología lineal para la gestión de luminarias de alumbrado público (Huang y Yuan, 2015) (Perandones, 2014) (Zhang y Li, 2017); sistema de control de luminarias para el ahorro del consumo de energía sin indicar cuál es el protocolo de la capa de aplicación utilizado (Sikder, Acar y Aksu, 2018); sistema de gestión de luminarias para hogares inteligentes que no incluye un cargador de baterías del nodo y que trabaja con IPv4 (Huang, Xiao, Liu y Lin, 2016), y sistema de gestión de luminarias que utiliza nodos sensores con paneles solares para la provisión de energía (Ardiansyah, Seungkyo y Fahmi, 2013).

Tomando en cuenta lo expuesto, es necesario evaluar la aplicabilidad del protocolo 6LoWPAN en las redes inalámbricas de sensores (Son y Quyet, 2015) empleadas en la gestión de luminarias en un hogar inteligente para desarrollar redes sensores y actuadores, escalables, flexibles, extensibles con nodos móviles (Hoang y Sindhu, 2016).

En este contexto, el objetivo de este estudio es evaluar la utilización de las redes inalámbricas de sensores para la gestión de las luminarias en un hogar con base en una red inalám- 
brica de sensores con 6LoWPAN, en una topología tipo estrella (Aravindh y Srevarshan, 2017); se utilizan herramientas estándares para la capa aplicación. El control de las luminarias emplea un dispositivo portátil basado en Android y conectado a la red internet con IPv6. La gestión de las luminarias implica encender, apagar, atenuar, detectar movimiento y temporizar el tiempo de encendido.

\section{Metodología}

En este estudio se implementa un prototipo de gestión de luminarias que cumple los siguientes requerimientos:

- Permite la gestión remota del encendido y apagado de las luminarias, control de la intensidad lumínica, temporización, detección de movimiento y simulación de presencia.

- La gestión remota se hace con dispositivos basados en Arduino, conectados a la red IPv6.

- El direccionamiento de los nodos sensores debe ser compatible con IPv6 y permitir una conectividad de extremo a extremo.

- Usa herramientas estándares para las comunicaciones en la capa aplicación de la arquitectura de la red inalámbrica de sensores.

\section{Componentes del prototipo}

El prototipo implementado (Figura 1) está formado por un dispositivo Android llamado cliente, una aplicación alojada en un computador llamada servidor, un nodo gateway, tres nodos sensores que forman parte de una red sensor inalámbrica que opera con IEEE 802.15.4 y la red IPv6, que se describen a continuación:

Figura 1. Diagrama del ambiente de prueba

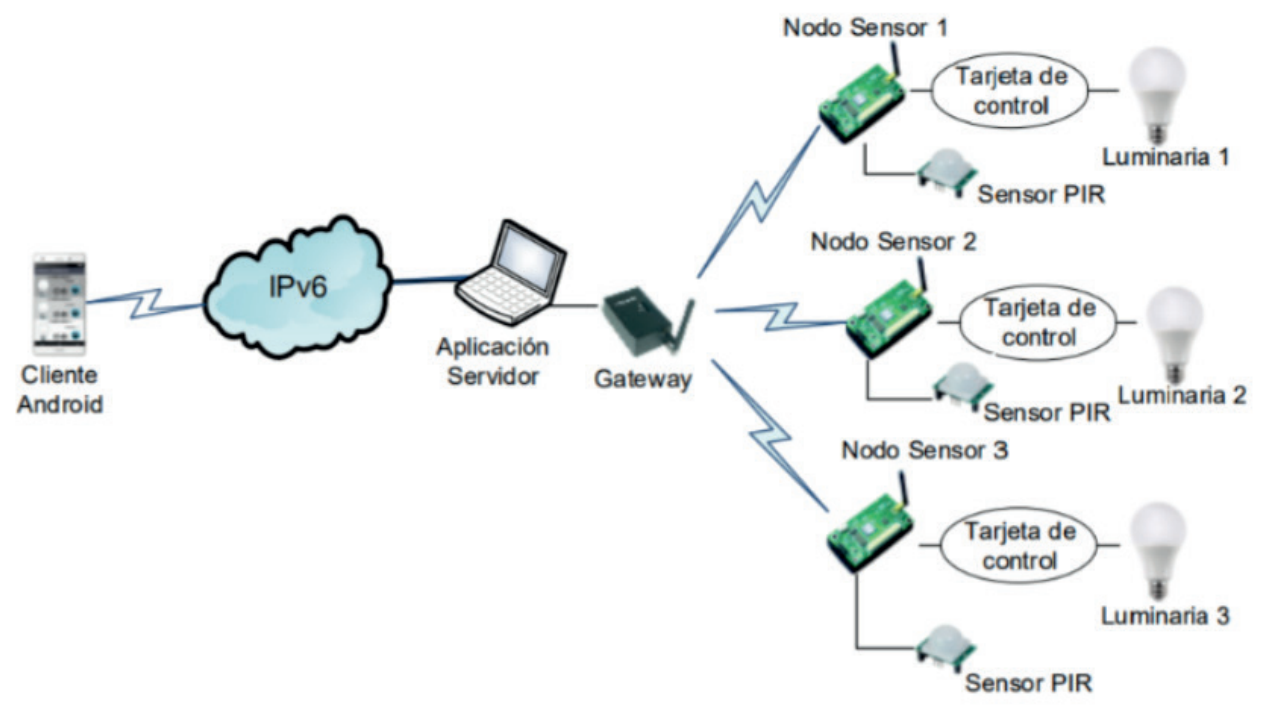




\section{Nodo sensor}

Es un nodo IRIS XM2110 (MEMSIC, 2012) (Figura 2) basado en un microcontrolador de baja potencia ATmega1281, de 8 bits, con un transmisor y receptor que operan en la banda $2.4 \mathrm{GHz}$. Este nodo gestiona el sensor de movimiento y la intensidad de la luminaria por medio de una tarjeta de control diseñada para tal efecto.

Figura 2. Nodo sensor IRISxm2110
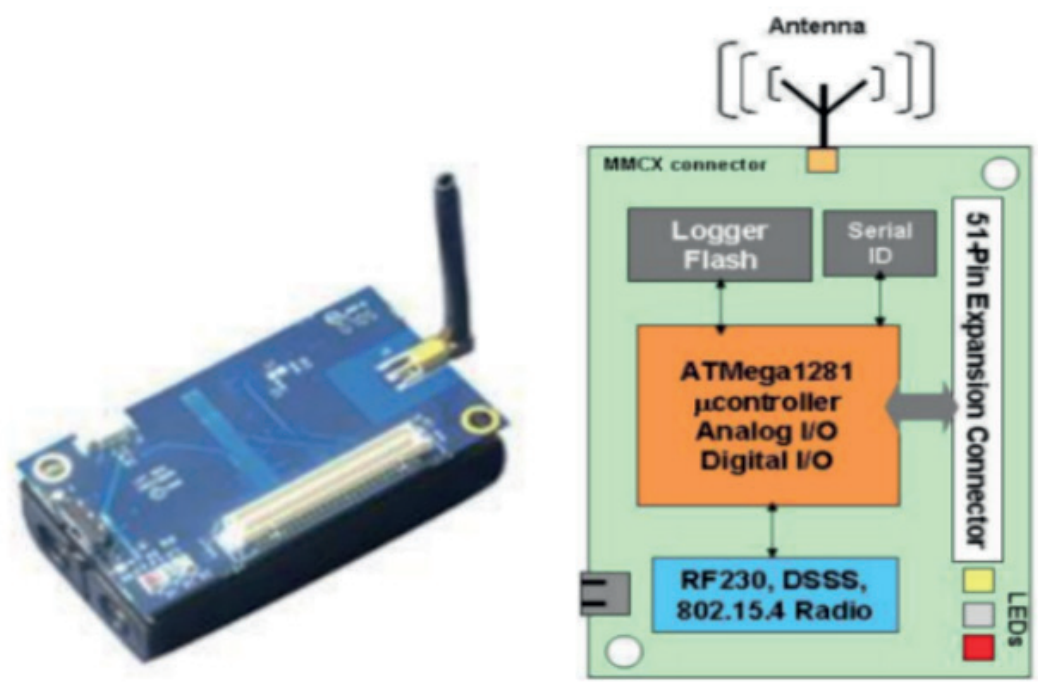

La información recolectada por cada nodo sensor se envía por medio del gateway hacia el servidor. El código de la aplicación fue creado con NesC y sus componentes se presentan en la Figura 3.

Figura 3. Componentes de la aplicación ejecutada en nodo sensor Iris XM21

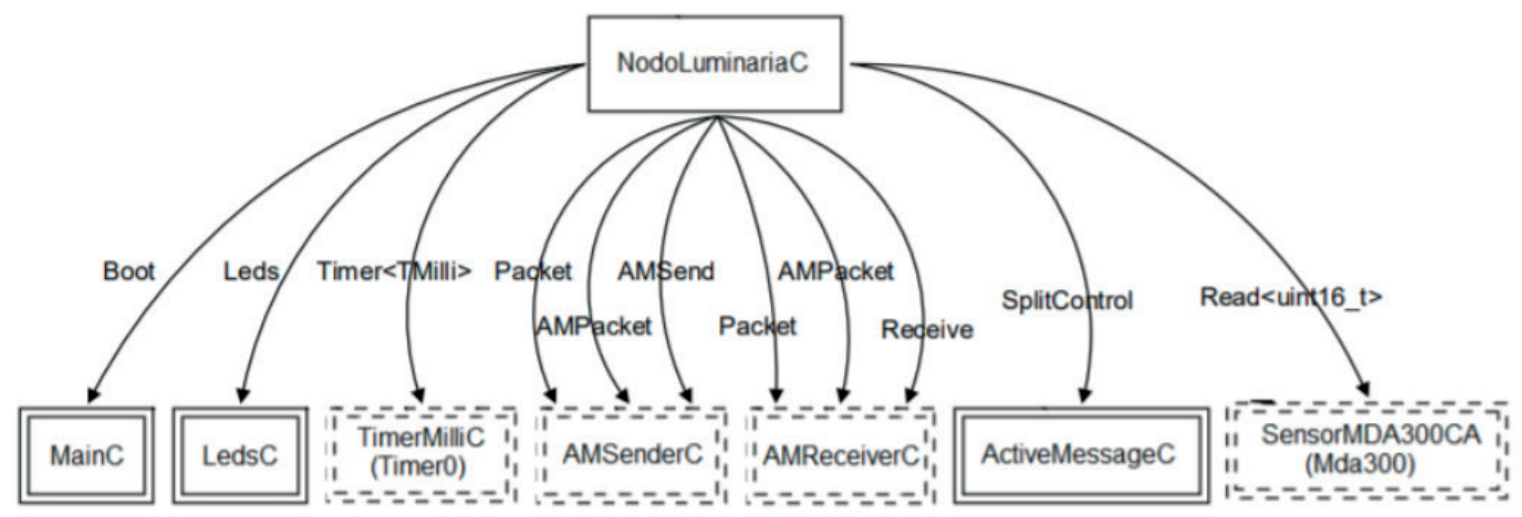

En la Figura 4 se presenta el componente de código nodoluminaria.h, en el cual se define la estructura del paquete, que consiste en la cabecera de 6LoWPAN con compresión HC1-HC2. Este paquete forma parte de la carga útil de la trama IEEE 802.15.4. 
Figura 4. Definición del direccionamiento para 6LowPAN

\begin{tabular}{|c|}
\hline $\begin{array}{l}\text { enum }\{ \\
\text { //Tipo mensaje Active Message } \\
\text { AM BLINKTORADIO }=6, \\
\text { //IIntervalos para tomar medidas } \\
\text { FRECUENCIA_MUESTREO }=10000\end{array}$ \\
\hline $\begin{array}{l}\text { \}; } \\
\text { typedef nx_struct BlinkToRadioMsg }\{ \\
\text { //Campos } 6 \text {-LoWPAN encapsulados } \\
\text { //en la trama IEEE } 802.15 .4 \\
\text { nx_uint64_t direccion_origen; } \\
\text { nx_uint64_t direccion_destino; } \\
\text { nx_uint8_t dispatch; } \\
\text { nx_uint8_t HC1; } \\
\text { nx_uint8_t limite_saltos; } \\
\text { nx_uint16_t datos; } \\
\text { \}-BlinkTo-_adioMsg; }\end{array}$ \\
\hline
\end{tabular}

\section{Tarjeta de control}

En la Figura 5 se presenta el dispositivo, implementado en este trabajo, acoplado al nodo sensor, el cual le permite al nodo sensor controlar el funcionamiento de la luminaria para encenderla, apagarla y controlar el nivel de iluminación.

Figura 5. Tarjeta de control

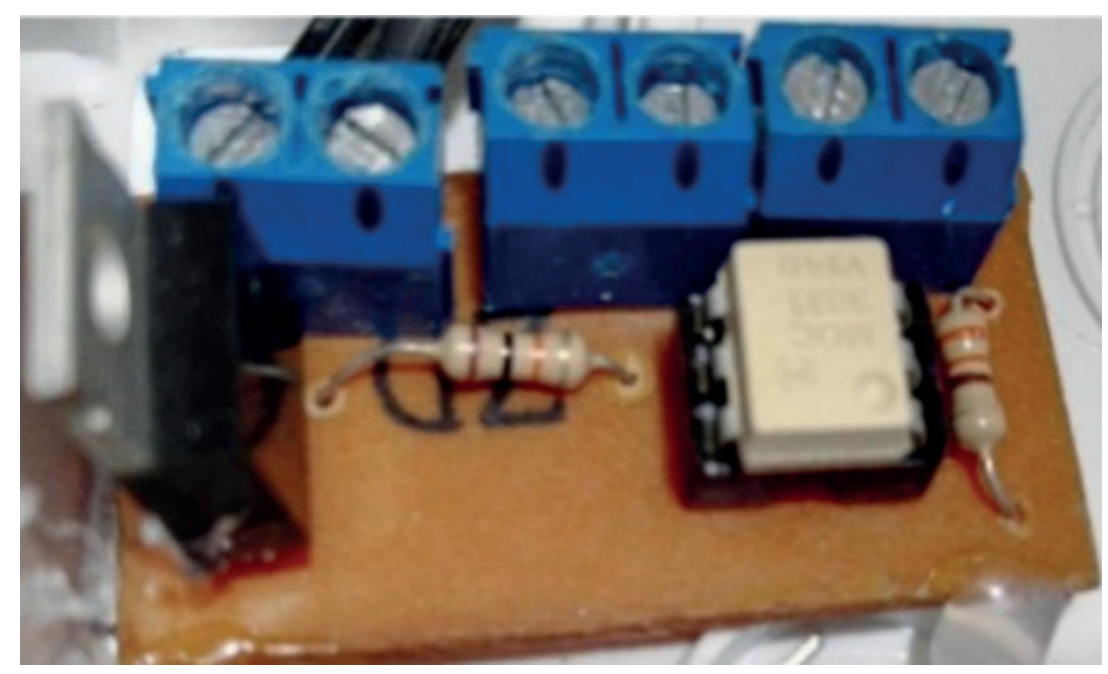

\section{Cliente Android}

Debido a que se necesita hacer la gestión remota utilizando una red IPv6, el cliente debe ser ejecutado en un dispositivo con el sistema operativo Android, con la versión 5.0 o superior, habilitado para tener conectividad con IPV6. Para desarrollar el aplicativo se utiliza el lenguaje de programación Java para implementar el sistema de gestión remota de las luminarias. En la Figura 6, se observa el diagrama de clases de la aplicación ejecutado en el cliente Android. 
Figura 6. Diagrama de clases de la aplicación Cliente

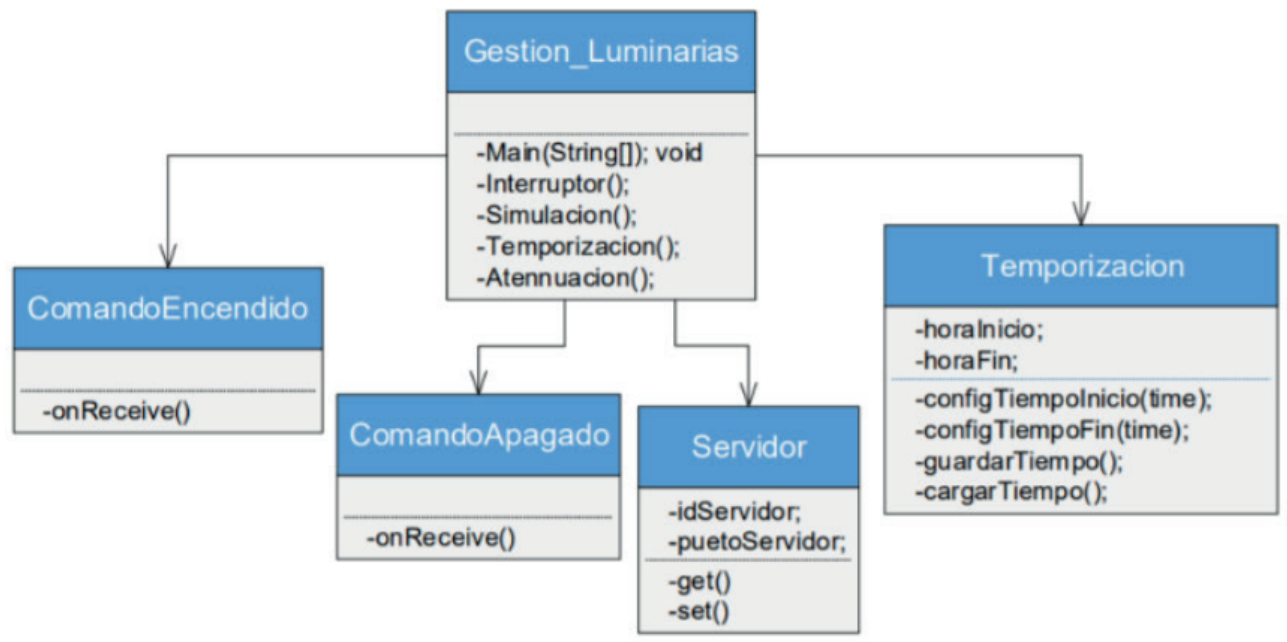

\section{Servidor}

Es el computador en el cual se ejecuta la aplicación Servidor para convertir los protocolos de la red sensor inalámbrica con 6LoWPAN al protocolo IPV6 de la red internet. Para hacer la conversión de protocolos, el servidor trabaja conjuntamente con un nodo sensor definido como Gateway, el cual se conecta al puerto serial del servidor. El servidor envía los datos a la aplicación Cliente usando el protocolo IPv6. La aplicación Servidor se implementa empleando lenguaje Java y el IDE Eclipse. El diagrama de clases se muestra en la Figura 7.

Figura 7. Diagrama de clases de la aplicación Servidor

\begin{tabular}{|c|c|}
\hline & NodoLuminariaMsg \\
\hline \multirow[b]{2}{*}{ Servidor } & $\begin{array}{l}\text {-DEFAULT_MESSAGE_SIZE; } \\
\text {-AM_TYPE; }\end{array}$ \\
\hline & -NodoLuminariaMsg(); \\
\hline+ MotelF motelF & $\begin{array}{l}\text {-get_direccion_origen(); } \\
\text {-set direccion origen(); }\end{array}$ \\
\hline $\begin{array}{l}\text {-Main(String[]); void } \\
\text {-Servidor(); } \\
\text {-enviarPaquetes(); } \\
\text {-recibirPaquetes(); } \\
\text {-SimulacionPresencia(); }\end{array}$ & $\begin{array}{l}\text {-get_direccion_destino(); } \\
\text {-set_direccion_destino(); } \\
\text {-get_dispatch(); } \\
\text {-set_dispatch(); } \\
\text {-get_HC1(); }\end{array}$ \\
\hline & $\begin{array}{l}\text {-set_HC1(); } \\
\text {-get_limite_saltos(); } \\
\text {-set_limite_saltos(); }\end{array}$ \\
\hline
\end{tabular}

El servidor envía los paquetes hacia el nodo Gateway y al dispositivo cliente. Los paquetes se capturan en el terminal del servidor usando las herramientas MsgReader (Sicos1977, 2013). Para visualizar los paquetes en el servidor se utilizó la herramienta Java Listen, que funciona como un monitor de paquetes (sniffer, en inglés) y facilita la presentación de los paquetes binarios en la pantalla. 


\section{Nodo gateway}

Implementado con el dispositivo MIB520 (MEMSIC, 2010), este nodo recolecta información de la red sensor inalámbrica que opera con el protocolo IEEE 802.15.4 y la transmite al servidor; además, recibe los datos desde el servidor y los retransmite hacia los nodos sensores para el control de las luminarias. Para las comunicaciones entre el servidor y los nodos sensores, el nodo Gateway utiliza la aplicación BaseStation (TinyOS, 2009), herramienta de TinyOS, que permite al Gateway operar como un puente entre el puerto serie del servidor y la red inalámbrica de sensores. Cuando el nodo Gateway recibe un paquete del puerto serie del servidor, lo transmite por radio; cuando recibe un paquete por la radio, lo transmite al puerto serie. De esta manera, la herramienta BaseStation establece la comunicación entre el servidor y los nodos sensores. La herramienta MIG (TinyOS, 2011) es empleada para encapsular el paquete en la carga útil de la trama IEEE 802.15.4; se pueden leer e imprimir los campos de la carga útil de la trama 802.15.4. Utilizamos la herramienta MsgReader (TinyOS, 2010) para extraer únicamente la carga útil de la trama omitiendo la cabecera IEEE 802.15.4.

\section{Red IPv6}

La red IPv6 se implementa con 3 ruteadores Cisco 1841 conectados en topología anillo y configurados con IPv6.

\section{Componentes del sistema}

Como se muestra en la Figura 8, inicialmente la aplicación Cliente Android, que se ejecuta en un teléfono celular, enviará una petición a la aplicación Servidor que opera con Ubuntu; estos dos componentes se comunicarán usando IPv6. Luego, el servidor envía un mensaje a la red inalámbrica IEEE 802.15.4 utilizando el Gateway. El Gateway y los nodos sensores operan con el sistema operativo TinyOS. En la misma figura, se aprecian las aplicaciones que se ejecutan en cada elemento que forma parte del prototipo. Con respecto a la manera en que se comunicarán los sistemas, las flechas indican la tecnología de comunicación empleada.

Figura 8. Componentes del sistema

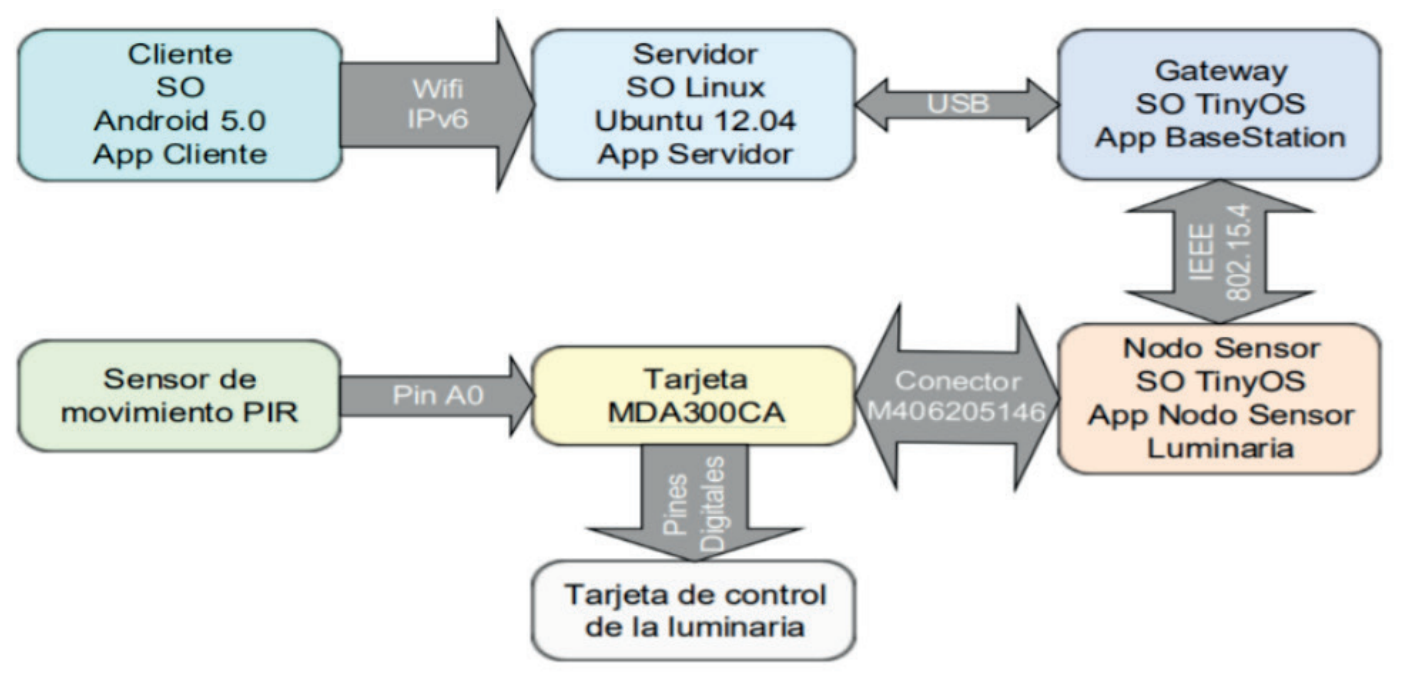




\section{Resultados y discusión}

La luminaria prototipo que se implementó para controlarla ser controlada en forma remota se observa en la Figura 9.

Figura 9. Luminaria implementada

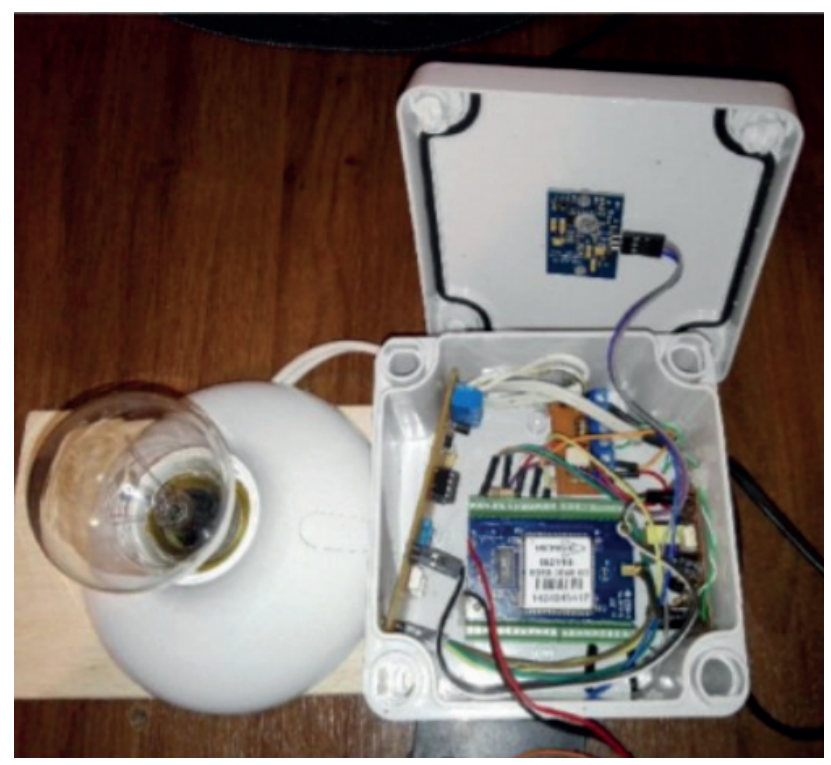

La aplicación desarrollada para el dispositivo con el sistema operativo Android (teléfono celular) tiene una interfaz (Figura 10) que permite activar y desactivar la temporización, elegir la hora que se enciende o se apaga la luminaria y presentar los datos de configuración del temporizador.

Figura 10. Interfaz para el control de la luminaria

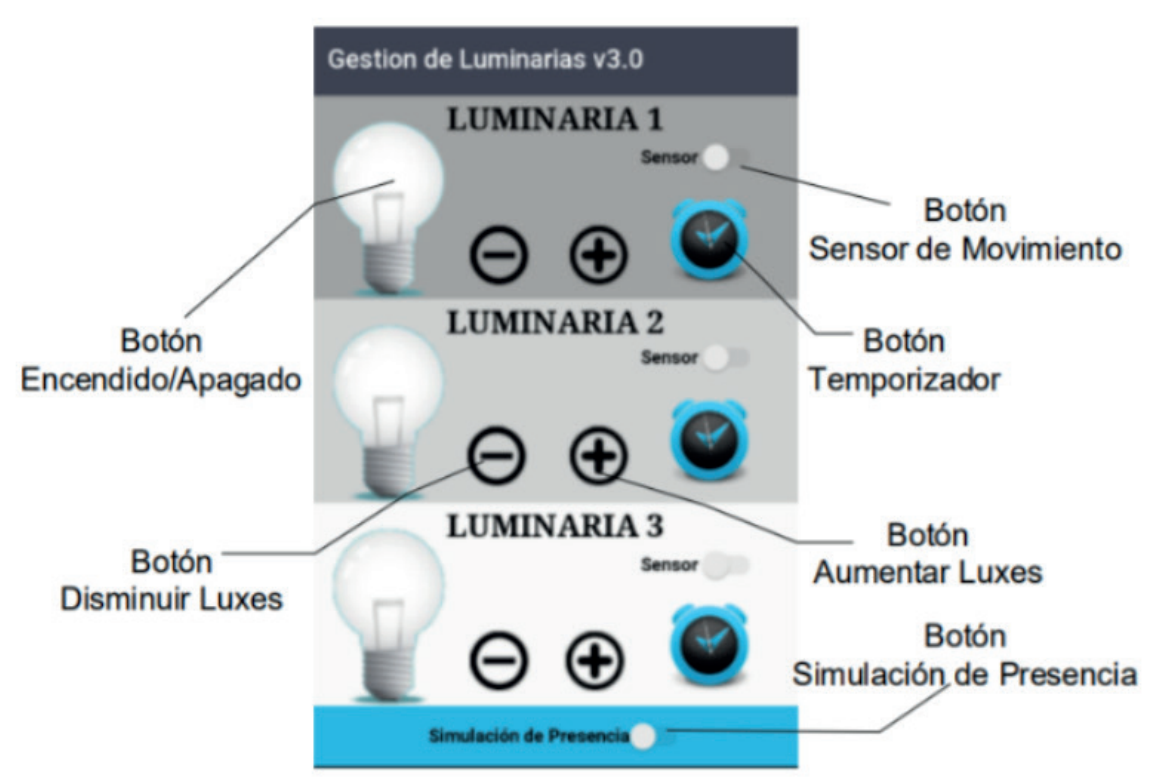


Configurados todos los componentes del prototipo, se validó el funcionamiento del sistema propuesto. Las funciones de encendido, apagado, temporización de la luminaria y control de la intensidad de iluminación funcionaron de acuerdo con los requerimientos de diseño.

En la Tabla 1, se presentan las direcciones IPv6 configuradas en los nodos sensores para hacer las pruebas de conectividad con el teléfono celular configurado con IPv6. El resultado de la función ping, que verifica la conectividad entre el nodo sensor y el teléfono celular, fue exitoso.

Tabla 1. Direcciones de los nodos sensores

\begin{tabular}{|l|l|}
\hline \multicolumn{1}{|c|}{ Columna 1 } & \multicolumn{1}{c|}{ Columna 2 } \\
\hline Gateway & FECO::1B \\
\hline Luminaria 1 & FECO::1 \\
\hline Luminaria 2 & FECO::2 \\
\hline Luminaria 3 & FECO::3 \\
\hline
\end{tabular}

En la Figura 11, se presenta la información del paquete 6LoWPAN enviado por el nodo sensor, capturado en el servidor, para ser convertido en un paquete IPv6 y recibido por el teléfono celular.

Para medir la intensidad de iluminación, se usó la aplicación basada en Android denominada 'luxómetro'; esta aplicación utiliza el sensor que está ubicado en la parte frontal del dispositivo celular.

Figura 11. Paquete 6LoWPAN recibido en el servidor

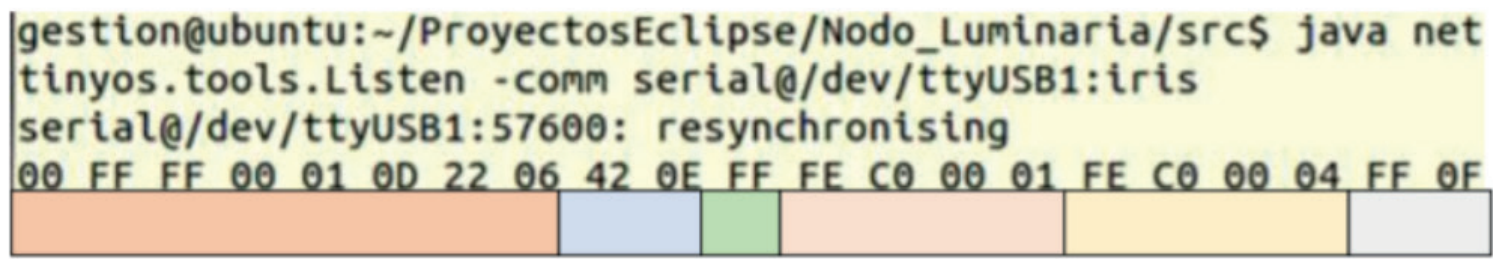

口 Cabecera IEEE 802.15.4

- Cabecera 6LoWPAN

- Límite de Saltos

Dirección Origen

Dirección Destino

口 Datos

\section{Control de luminosidad}

Se obtuvieron las medidas de intensidad de luz de la luminaria utilizando el luxómetro (ver Tabla 2); la disminución y el aumento de la intensidad de luz medida permitieron comprobar el control sobre la luminaria desde el teléfono celular. La intensidad de luz medida depende del voltaje suministrado a la luminaria y de los luxes del ambiente. La variación de lúmenes medidos al pasar de un nivel de intensidad a otro no es fija, debido al tipo de foco y a la variación del voltaje que tiene un valor promedio de 13 voltios. 
Tabla 2. Medidas obtenidas con el luxómetro

\begin{tabular}{|l|l|l|l|l|}
\hline $\begin{array}{c}\text { Número } \\
\text { de Medida }\end{array}$ & \multicolumn{1}{|c|}{$\begin{array}{c}\text { Ambiente } \\
\text { (Luxes) }\end{array}$} & \multicolumn{1}{|c|}{$\begin{array}{c}\text { Luxómetro } \\
\text { (Luxes) }\end{array}$} & $\begin{array}{c}\text { Ambiente } \\
\text { (Luxes) }\end{array}$ & $\begin{array}{c}\text { Luxómetro } \\
\text { (Luxes) }\end{array}$ \\
\hline 1 & 146 & 4100 & 79 & 82 \\
\hline 2 & 175 & 3595 & 104 & 466 \\
\hline 3 & 116 & 2739 & 91 & 905 \\
\hline 4 & 152 & 554 & 115 & 3278 \\
\hline 5 & 110 & 110 & 146 & 3486 \\
\hline
\end{tabular}

\section{Temporización}

En la Tabla 3, se presentan los resultados de la prueba de la temporización, en la cual se pueden apreciar los tiempos de respuesta del control de encendido de la luminaria en forma remota.

Tabla 3. Medidas de tiempos de temporización

\begin{tabular}{|l|l|l|l|}
\hline \multicolumn{2}{|c|}{ Tiempo configurado } & \multicolumn{2}{c|}{ Tiempo medido } \\
$\begin{array}{c}\text { Hora } \\
\text { Encendido }\end{array}$ & \multicolumn{1}{|c|}{$\begin{array}{c}\text { Hora } \\
\text { Apagado }\end{array}$} & \multicolumn{1}{c|}{$\begin{array}{c}\text { Hora } \\
\text { Encendido } \\
\text { Apagado }\end{array}$} \\
\hline $18: 00: 00$ & $21: 00: 00$ & $18: 00: 23$ & $21: 00: 35$ \\
\hline $13: 15: 00$ & $13: 45: 00$ & $13: 15: 08$ & $13: 45: 40$ \\
\hline $19: 34: 00$ & $22: 30: 00$ & $19: 34: 21$ & $22: 30: 51$ \\
\hline $09: 40: 00$ & $11: 00: 00$ & $09: 40: 05$ & $11: 00: 02$ \\
\hline
\end{tabular}

A pesar de que los resultados obtenidos con la función de temporización fueron satisfactorios, cabe mencionar que las acciones de prendido y apagado de la luminaria no fueron ejecutadas de manera inmediata y en el momento indicado, sino que se llevaron a cabo un tiempo después de la hora definida. Esto se debe a que en el celular se estaban ejecutando otras aplicaciones, y el servicio utilizado para el envío de mensajes no tiene una prioridad alta.

\section{Simulación de presencia}

Durante esta prueba se verificó que el encendido y apagado de la luminaria de manera aleatoria. Se hicieron pruebas con franjas horarias y se constató el funcionamiento correcto. Los resultados obtenidos cumplen con la función de simulación de presencia codificada.

\section{Discusión}

La mayoría de implementaciones hechas para la gestión de luminarias utilizan protocolos propietarios de comunicaciones en la capa aplicación (Han et al., 2010) (Park y Hong, 2010) (Wohlers et al., 2013) (Domínguez et al., 2012), debido a que las empresas fabricantes quieren mantener el liderazgo en el mercado, y porque no están definidos los estándares para la capa aplicación de la red inalámbrica de sensores (Rand, 2018). Por esta razón, los fabricantes restringen el 
acceso al código y a las herramientas para su implementación. En el trabajo se evidencia la utilización de herramienta y aplicativos de comunicaciones, a nivel de capa aplicación, de libre acceso para implementar el sistema de gestión de luminarias, lo cual es el aporte fundamental de este estudio.

Asimismo, la implementación de luminarias gestionadas en forma remota implica que la provisión de energía, por medio del cable de energía eléctrica, es la misma para la luminaria y para el nodo sensor, debido a que este nodo está acoplado a la luminaria. En el prototipo propuesto se ha incluido la utilización de un sistema de provisionamiento de energía independiente de la red eléctrica, lo cual permite la gestión de la luminaria por el nodo gestor, aun cuando no exista la provisión de energía para la luminaria y para el nodo sensor, escenario que se produce cuando la luminaria no recibe energía eléctrica.

Finalmente, se considera que el futuro de la gestión de luminarias para hogares inteligentes está relacionado con los nodos sensores de bajo consumo de energía, y que tengan una autonomía en los sistemas de provisión de energía de la red eléctrica para su funcionamiento con la implementación de nodos sensores de tamaño reducido.

\section{Conclusiones y recomendaciones}

La utilización de nodos sensores de baja capacidad de procesamiento es una opción óptima para implementar redes sensores y actuadores para la gestión remota de luminarias empleando los protocolos estándar IPV6 y 6LoWPAN.

La asignación de direcciones públicas a los nodos sensores facilita la conectividad de extremo a extremo en comparación con otros protocolos de red.

La conectividad entre la red sensor inalámbrica y la red internet siempre necesitará de un Gateway de alta capacidad de procesamiento que se utiliza para la conversión de protocolos.

La complejidad de la implementación de 6LoWPAN en los nodos sensores puede minimizarse haciendo en el Gateway los procesos 6LoWPAN que corresponde a los nodos sensores.

Se han empleado herramientas de desarrollo de acceso libre para implementar el prototipo, el cual sirve como base para el desarrollo de aplicaciones para el Internet de Todas las Cosas utilizando redes inalámbricas de sensores.

Finalmente, se recomienda trabajar en un protocolo para la capa aplicación en redes inalámbricas de sensores y, de esta manera, promover el desarrollo de nuevas aplicaciones escalables y compatibles.

\section{Bibliografía}

Al-Kashoash. (2017). Comparison of 6LoWPAN and LPWAN for the Internet of Things. Australian Journal of Electrical and Electronics Engineering, 268-274.

Aravindh, J. y Srevarshan, B. (2017). Home Automation in loT Using 6LowPAN. Advanced Computational Engineering and Networking, 26-28.

Ardiansyah, E.; Seungkyo, O., y Fahmi, A. (2013). Battery-Less 6LoWPAN-Based Wireless Home Automation by Use of Energy Harvesting. International Journal of Distributed Sensor Networks.

Domínguez, F.; Touhafi, A., y Tiete, J. (2012). Coexistence with WiFi for a Home Automation ZigBee Product. $19^{\text {th }}$ Symposium on Communications and Vehicular Technology in the Benelux (SCVT), 1-6. Eindhoven: IEEE. 
Han, N.; Wu, Y, y Tang, Y. (2010). Research of KNX Device Node and Development Based on the Bus Interface Module. $29^{\text {th }}$ Chinese Control Conference (CCC), 4346-4350.

Hoang, D. y Sindhu, S. (2016). A Wireless Sensor and Actuator Network (WSAN) Framework for Personalized Thermal Comfort in Office Buildings. International Conference on Sustainable Energy Technologies. Vietnam: IEEE.

Huang, L.; Xiao, L.; Liu, J., et al. (2016). LED Intelligent Lighting System Based on 6LoWPAN. International Conference on Computer Science \& Education. Nagoya: IEEE.

Huang, Z. y Yuan, F. (2015). Implementation of 6LoWPAN and Its Application in Smart Lighting. Journal of Computer and Communications, 3, 80-85.

IEEE. (2011). Part 15.4: Low-Rate Wireless Personal Area. New York: IEEE Computer Society.

MEMSIC. (2010). Powerful Sensing Solutions. MEMSIC. Recuperado de http://www.memsic.com/userfiles/files/datasheets/wsn/6020-0091-04_a_mib520cb-t.pdf

MEMSIC. (2012). Document Part Number: 6020-0124-01 Rev A. MEMSIC. Recuperado de www.mensic. com: http://www.memsic.com/userfiles/files/Datasheets/WSN/IRIS_Datasheet.pdf

Park, T., y Hong, S. (2010). A New Proposal of Network Management System for BACnet and Its Reference Model. $8^{\text {th }}$ IEEE International Conference on Industrial Informatics (INDIN), 28-33. Osaka: IEEE.

Perandones, M. (2014). Energy-saving Smart Street Lighting System based on 6LoWPAN. Proceedings of the First International Conference on loT in Urban Space, 93-95

Rand, P. (2018). Wireless Lighting Control: The Bright Road Ahead., Strategic marketing. Recuperado de https://m.eet.com/media/1118195/ ti\%20paper.pdf

Sicos K.. (2013). MSGReader. GitHub. Obtenido de https://github.com/ Sicos1977/ MSGReader

Sikder, A.; Acar, A., y Aksu, H. (2018). IoT-enabled Smart Lighting Systems for Smart Cities. $8^{\text {th }}$ Annual Computing and Communication Workshop and Conference. Las Vegas: IEEE.

Singh, M. (2016). Wireless Sensor Networks: A Survey. Journal of Scientific \& Engineering Research, 7(4), 710-716.

Sohraby, K.; Minoli, D., y Znati, T. (2007). Wireless Sensor Networks, Technology, Protocols, and Applications. New Jersey, Estados Unidos: John Wiley \& Sons.

Son, H. y Quyet, C. (2015). Design, Implementation, and Evaluation of 6LoWPAN for Home and Building Automation in the Internet of Things. $12^{\text {th }}$ ACS/IEEE International Conference on Computer Systems and Applications. Morocco: IEEE.

TinyOS. (2009). BaseStation. GitHub. Recuperado el de github: https://github.com/tinyos/tinyos-main/ tree/master/apps/BaseStation

TinyOS. (2010).Mote PC serial communication and SerialForwarder. TinyOs-Stanford http://tinyos.stanford.edu. Recuperado el de http://tinyos.stanford.edu/tinyos-wiki/index.php/Mote_PC_serial_ communication_and_SerialForwarder_(TOS_2.1.1_and_later

Wohlers, I.; Andonov, R., y Gunnar, K. (2013). DALIX: Optimal DALI Protein Structure Alignment. IEEE/ACM Transactions on Computational Biology and Bioinformatics, 10(1), 26-36.

Zhang, Q. y Li, P. (2017). Design and Implementation of an Intelligent Street Lamp Network Node Based on 6LoWPAN Technology. International Conference on Computer, Electronics and Communication Engineering. Sanya: IEEE. 